Ann. Génét. Sél. anim., 1981, 13 (4), 339-352

\title{
Possible formule génétique pour la couleur du pelage de la race bovine Brune des Alpes
}

\author{
J.J. LAUVERGNE \\ I.N.R.A., Laboratoire de Génétique factorielle, Centre de Recherches zootechniques \\ F-78350 Jouy-en-Josas
}

\begin{abstract}
Résumé
Jusqu'à présent, on avait considéré que la couleur du pelage de la race Brune des Alpes était déterminée par l'allèle $A^{w}$ au locus $A$ gouti, les autres loci portant les allèles non mutés, en particulier $B^{+}$en $B$ (Brun) qui aurait induit de l'eumélanine noire. On pense maintenant qu'il faut mieux adopter une formule avec $A^{i}$ ( $i$ pour raie de mulet inversée) au locus Agouti et $B^{s}$ ( $s$ pour brun chocolat schwyz) au locus $B$. Le phénotype "raie de mulet inversée » (corps noir, raie de mulet inversée claire ou rouge, intérieur des oreilles rouge, tour du mufle clair) se rencontre en effet aussi sur fond eumélanique noire et la couleur du corps de la Brune des Alpes présente les mêmes nuances que certains ovins ou caprins dont l'eumélanine est brun chocolat.
\end{abstract}

\section{I. - Introduction}

La génétique de la coloration du pelage est une discipline qui progresse lentement dans l'espèce bovine comme le prouve, par exemple, l'article récent d'OLson (1980) où il s'agit encore de déterminer quel pourrait être le phénotype sauvage de l'espèce.

Les raisons en sont en premier lieu les difficultés d'expérimentations dues au coût élevé de chaque sujet et à la longueur de l'intervalle de génération. De plus, les données recueillies en ferme comprennent surtout des $F_{1}$ et peu de back cross ou $F_{2}$ et l'interprétation de résultats en provenance de lignées multigrégantes comme celles de WiLson (1909) pose plus de problèmes qu'elle n'aide à en résoudre.

Dans ces conditions on ne peut espérer progresser que si l'on dispose d'un modèle tiré de l'étude d'autres espèces et qui peut être adapté à l'espèce bovine.

Il se trouve qu'un tel cadre existe pour tous les Mammifères comme l'avait déjà pressenti Sewall Wright à la fin de la deuxième décennie de ce siècle dans une série d'articles restés célèbres (cf. SEARLe, 1968 pour l'exposé historique) : c'est celui de l'homologie de certains loci pour la synthèse et la distribution, dans le pelage, des seuls pigments existant à ce niveau: les mélanines. Cette homologie, mise en évidence tout d'abord dans des espèces expérimentales ou domestiques de rongeurs 
(souris, rat, cobaye...) et carnivores (chat, chien...) (cf. Haldane, 1927 et LitTle, 1958) se vérifie également pour des espèces de ruminants proches des bovins : les ovins (Adalsteinsson, 1970, 1974 ; Lauvergne \& Adalsteinsson, 1976) et les caprins (LAUVergne, 1978 b ; LaUVERGNe \& Howell, 1978). Elle est spécialement frappante par les loci de coloration proprement dits (ceux qui contrôlent biochimiquement la synthèse pigmentaire dans les mélanocytes) : Agouti (A), Brun (B), Albinisme vrai (C) et Extension du Noir (E), cf. Prota \& Searle (1978).

Pour les bovins, toutefois, si WRIGHT (1917) avait déjà proposé le fil directeur de l'homologie, il n'a, pendant un demi-siècle, été que très partiellement suivi - à l'exception toutefois de CaStLE $(1931,1940)(1)$ - cependant que des interprétations beaucoup plus «libres» obtenaient une audience plus grande. Ainsi Sмiтh (1924) aux Etats-Unis a une conception assez personnelle qui sera néanmoins reprise et amplifiée par IBSEN (1933) lequel, à son tour, trouvera des émules en la personne de SHrode \& Lusch (1946) et surtout de Berge en Norvège (1949, 1961 b et 1965). Avec StafFe (1925) naît en Autriche un autre modèle interprétatif assez différent de celui des Américains cités précédemment mais qui n'en aura pas moins un succès durable auprès des auteurs de langue allemande comme WiETHE-KoERPRICH (1952) et Kliesch \& Horst (1960) (cf. Lauvergne, 1966 a, pour un exposé plus détaillé de l'historique de ces interprétations).

En fait, dans les années soixante, on n'avait aucun mal à démontrer le manque de réalisme de telles interprétations. C'est ainsi que SMITH (1924) fait intervenir 6 loci et IBSEN (1933) 8 loci pour la coloration proprement dite et qu'avec BERGE (1965) ce nombre dépassera la dizaine, tous ces loci ne correspondant à ceux bien isolés par l'homologie que par hasard. La pratique de la juxtaposition avec SHrode \& LusH (1946) qui ajoutent C (Albinisme vrai), RENDEL (1957) E (Extension), BERGE (1956) A (Agouti), aboutit à une confusion qui culmine chez BERGE (1961 b et 1965).

Pour repartir sur des bases saines nous avons pensé qu'il valait mieux faire table rase sur ces interprétations pour ne retenir que les allèles présentant des caractères d'homologie dans leur comportement avec d'autres espèces et ainsi groupables dans les principales séries admises généralement chez les Mammifères pour le contrôle de la couleur du pelage. Pour les loci de coloration proprement dits on a proposé de cette manière les 3 séries: $A, C$ et $E$ avec ladjonction de $D$ la Dilution Maltaise (LAUVERGNe, 1966 a). Cette interprétation a été reconnue comme plus réaliste par Searle (1968) et adoptée par Adalsteinsson dans sa revue de 1974.

Plus récemment nous avons introduit quelques modifications à cette interprétation. Tout d'abord, dans une note parue en 1977, nous avons ajouté au locus Agouti les allèles $A^{g}:$ gris du Gascon (2) (en supprimant l'allèle chinhilla $C^{c h}$ en $C$ ) $A^{t}$ (noir et feu, précédemment isolé chez des zébus de Madagascar, LAUVERGNE, 1970) et $A^{u}$ (blaireau: le blackish pattern décrit par BALDwIN et al., 1956, en race Jersey d'Amérique) tout en conservant $A^{y}$, fauve à extrémités noires, $A^{+}$, agouti dun, et $A^{w}$, ventre blanc (ou white belly), caractérisant la Brune des Alpes. De ce locus nous écartions alors $A^{8}$ (self, noir uniforme) qui nous semblait plutôt, d'après sa dominance même, devoir

(1) Ce qui s'explique par une filiation intellectuelle puisque Wright a été l'élève de CaSTLE. Par ailleurs toutefois CASTLE n'a pas eu l'occasion d'analyser des données de ségrégation de la couleur chez les Bovins.

(2) Ce phénotype se rencontre également en Grise des steppes de Hongrie et chez toutes les races d'Italie dites podoliques : Chianine, Piémontaise, Romagnole... 
être placé au locus d'Extension en tant que $E^{d}:$ noir dominant comme l'avait suggéré RENDEL (1957).

Dans une note suivante (Lauvergne, 1978 a) nous pensions qu'il était plus rationnel de placer le rouge acajou uniforme (type Salers) au locus Agouti (déterminé par $A^{r}$ ) plutôt qu'au locus d'Extension $\left(E^{r}\right)$ cependant que $A+$ (agouti dun) de LAUVERgne (1966 a) était scindé en deux : $\boldsymbol{A}^{+}$l'allèle sauvage (voisin de $\boldsymbol{A}^{y}$ ) et $\boldsymbol{A}^{d}$ le $d u n$, ce qui correspond à la conception avancée plus tard par OLSON (1980), sans que cet auteur ait eu connaissance de notre article. Pour terminer l'exposé des modifications proposées dans cette note il faut mentionner l'introduction d'un nouvel allèle au locus $A g o u t i, A^{l}$ : fauve clair (1 pour light) pour interpréter la coloration de races de type Gelbvieh, Blonde d'Aquitaine ou Villard de Lans.

Cette évolution de nos conceptions qui est présentée dans le tableau 1 (colonnes 2 et 3) se caractérise principalement par un allongement de la série Agouti. Elle a été inspirée par l'observation des systèmes colorés des ovins et caprins dont les progrès au cours de la décennie 1970-1980 ont été évoqués précédemment. Cette polyallélie de la série Agouti est d'ailleurs observée dans des espèces de Mammifères mieux étudiés (par exemple la souris, Silvers, 1980).

Ces tâtonnements et retours en arrière mettent en évidence la fragilité de certaines hypothèses. Ils indiquent que beaucoup d'études sont encore nécessaires.

Dans le présent article, nous avons réuni des faits et des arguments afin de reconsidérer la formule colorée de la race Brune des Alpes.

\title{
II. - Matériel et méthodes
}

\author{
A. - La Brune des Alpes, sa création, son extension \\ ses appellations et son standard coloré
}

La création officielle en Suisse de la Brune Suisse ou Brune des Alpes remonte à 1869. En 1880 c'est une race déjà bien établie dans son pays d'origine et elle constitue entre 1886 et 1956 autour de $45 \%$ du cheptel national, étant cantonnée en gros dans la moitié Est du pays (le Herd book a son siège à Zoug) (cf. ENGELER, 1947 et Bonadonna, 1959).

Très tôt cette race rayonne dans le monde entier (dès 1911 par exemple il existe un herd book Brun des Alpes en France, QuitTET et Denis, 1979) où elle prend des noms divers qui rappellent sa couleur: Brune en français, Graubraun (gris brun) en allemand, Brown en anglais, Bruna en italien, etc.; son origine montagneuse : Brune des Alpes en français, Brown Mountain en anglais, Graubraunes Höhenvieh en allemand) ou, simplement géographique: race $S c h w y z$ en français, Brown Swiss en anglais et son pays d'adoption: American Brown Swiss par exemple (cf. MASON, 1956 pour toutes ces questions de nomenclature raciale). 
Tableau 1

Interprétations alléliques successives de l'auteur à 3 des loci de coloration proprement dits des bovins

Successive allelic interpretation of the author for 3 of the basic loci for cattle coloration

\begin{tabular}{|c|c|c|c|c|}
\hline \multirow[b]{2}{*}{ Loci } & \multicolumn{4}{|c|}{ Séries alléliques selon } \\
\hline & $\begin{array}{c}\text { LAUVERGNE (1966) (a) } \\
1\end{array}$ & $\begin{array}{c}\text { LAUVERGNE (1977) } \\
2\end{array}$ & $\begin{array}{c}\text { LAUVERGNE } \\
\text { (1979) } \\
3\end{array}$ & $\begin{array}{l}\text { LAUVERGNE } \\
\text { (présent } \\
\text { article) } \\
4\end{array}$ \\
\hline $\begin{array}{l}\text { A } \\
\text { Agouti } \\
(1)\end{array}$ & 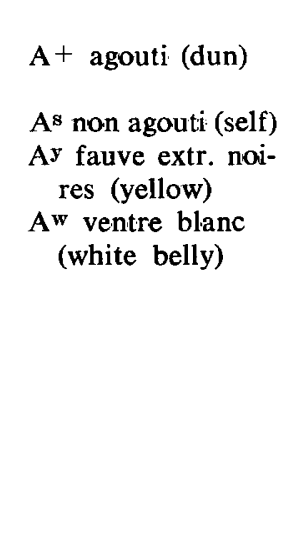 & $\begin{array}{l}\mathrm{A}^{+} \\
\mathrm{A}^{\mathbf{y}} \\
\mathrm{A}^{\mathrm{w}} \\
\mathrm{Ag}^{\mathrm{g}} \text { gris } \\
\mathrm{A}^{\mathrm{t}} \text { noir et feu (tan) } \\
\mathrm{A}^{\mathrm{b}} \text { blaireau } \\
\mathrm{A}^{1} \text { fauve clair } \\
\text { (light) }\end{array}$ & $\begin{array}{l}\begin{array}{c}\mathrm{A}+\text { sauvage } \\
\text { (non muté) }\end{array} \\
\mathrm{A}^{\text {d dun }} \\
\mathrm{A}^{\mathbf{y}} \\
\mathrm{A}^{\mathbf{w}} \\
\mathrm{A}^{\mathrm{g}} \\
\mathrm{A}^{\mathbf{t}} \\
\mathrm{A}^{\mathbf{b}} \\
\mathrm{A}^{\mathrm{I}} \\
\mathrm{A}^{\mathbf{r}} \text { rouge }\end{array}$ & $\begin{array}{l}A^{+}(2) \\
A^{d}(2) \\
A^{s} \\
A^{y} \\
\\
A^{t} \\
A^{b} \\
A^{1}(2) \\
A^{r} \\
A^{i} \text { raie de mu- } \\
\quad \text { let inversée }\end{array}$ \\
\hline $\begin{array}{c}\text { B } \\
\text { Brun } \\
(3)\end{array}$ & $\mathrm{B}+$ sauvage & B+ & $\mathbf{B}+$ & $\begin{array}{l}\text { B+ } \\
\text { Bs schwyz }^{\text {s sches }}\end{array}$ \\
\hline $\begin{array}{c}\mathrm{E} \\
\text { Extension } \\
(3)\end{array}$ & $\begin{array}{l}E+\text { sauvage } \\
E^{\text {br bringé }} \\
\mathrm{E}^{\mathrm{r}} \text { rouge }\end{array}$ & $\begin{array}{l}E^{d} \text { noir dominant } \\
E^{+} \\
E^{\text {br }}(2) \\
E^{r}(2)\end{array}$ & $\begin{array}{l}E^{d}(2) \\
E^{+} \\
E^{b r}(2)\end{array}$ & $\begin{array}{l}E^{d}(4) \\
E^{+} \\
E^{\text {br }}(2)\end{array}$ \\
\hline $\begin{array}{l}\text { D } \\
\text { Dilution } \\
\text { (3) }\end{array}$ & $\begin{array}{l}\mathrm{D}^{\mathrm{f}} \text { dilution } \\
\quad \text { fleckvieh } \\
\mathrm{D}+\text { sauvage }\end{array}$ & $\begin{array}{l}D^{f}(2) \\
D+(2)\end{array}$ & $\begin{array}{l}D^{f}(2) \\
D+(2)\end{array}$ & $\begin{array}{l}D^{f}(2) \\
D+(2)\end{array}$ \\
\hline
\end{tabular}

(1) Les allèles ne sont pas classés selon l'ordre de dominance. The alleles are not ordered according the dominancy.

(2) Allèle dont l'existence est ou a été tacitement reconduite. Allele whose existence is or has been tacitly admitted.

(3) Ces allèles sont classés selon le probable ordre de dominance du haut vers le bas du tableau.

The alleles are classified according the probable order of dominance from above to the bottom of the table.

(4) Allèle dont l'existence est remise en question. Allele whose existence is questioned. 
Le standard coloré est donné en détail par QuiTTET \& Denis (1979). On y relève les points suivants (cf. fig. 1 a) :

- un manteau avec des nuances diverses, variant du gris argenté et du brun foncé au brun clair: le gris souris étant la couleur préférée;

- un mufle ardoisé entouré de brun ou de gris très clair presque blanc;

- l'intérieur des membres d'une couleur jaunâtre ;

- une ligne dorsale parcourue par une raie de nuance claire (pas toujours présente d'ailleurs).
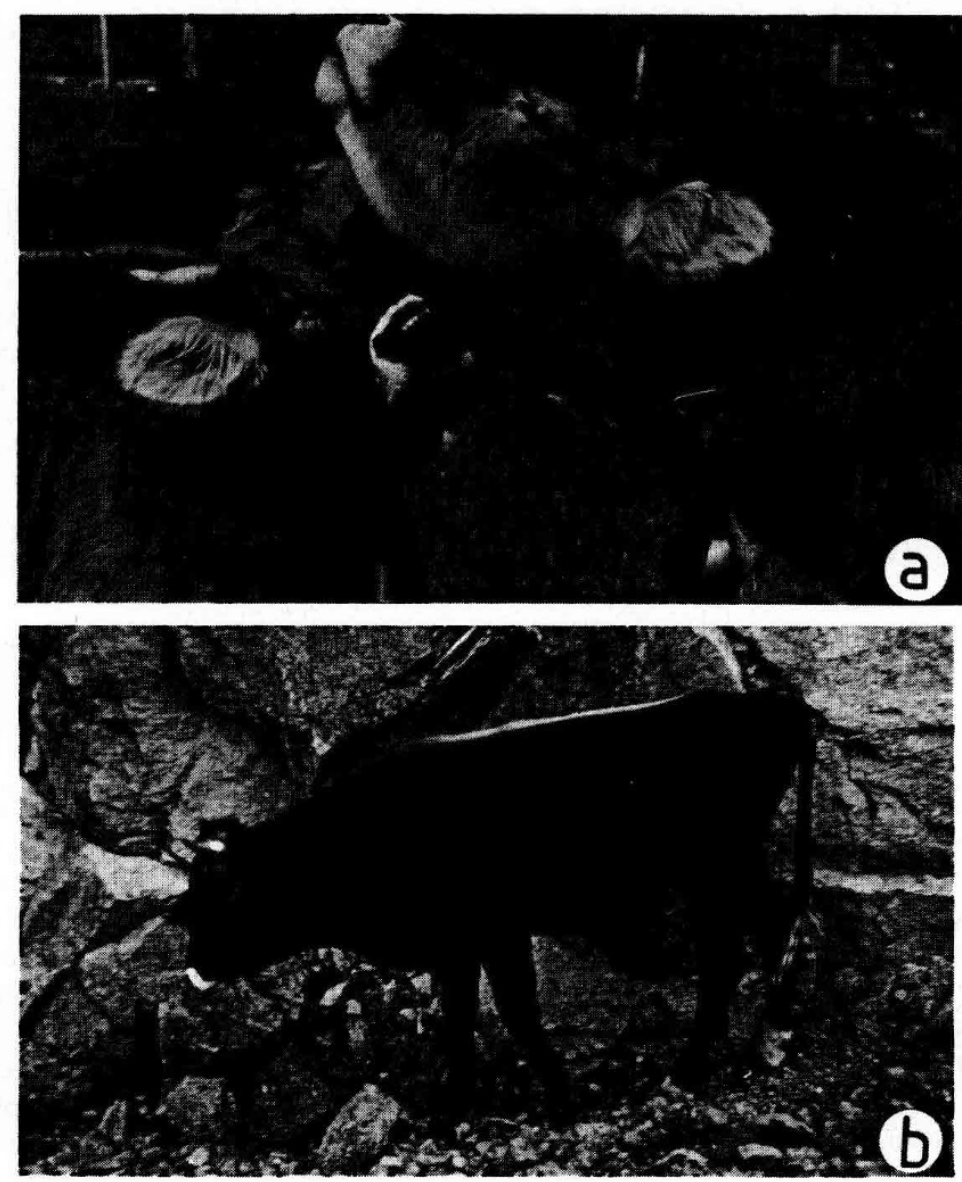

FIG. 1

a) Bovins Bruns des Alpes. On note la raie de mulet inversée, le tour du museau clair ainsi que les éclaircissements des oreilles.

b) Bovin Corse présentant le phénotype «raie de mulet inversée» sur fond eumélanique noir.

a) Alp brown cattle. Note the reversed mule stripe, the muzzle ring and the clear insides of ears.

b) Corsican native cattle with "reversed mule stripe» phenotype on black eumelanic background. 


\section{B. - Les formules génétiques successivement proposées pour la couleur de la race Brune des Alpes}

Depuis Staffe (1925) les auteurs de langue allemande comme WiEThE-KoERPRich (1952) et KLIESCH \& Horst (1960) s'en sont tenus à la formule suivante :

$$
\begin{array}{llllll}
k & k & B & B & G & G
\end{array}
$$

Avec $k$ au locus $K$ pour inhiber la manifestation de la couleur rouge ou noire, $B$ au locus $B$ pour induire le rouge ( $b=$ jaune) et $G$ au locus $G$ pour donner le gris (en allemand on dit «grau» ou «graubraun» pour la couleur de la race) qui domine $g$ : absence de gris.

Pour Berge (1956 et 1961 a) la couleur de la Brune des Alpes, quil qualifie en anglais de «dun brown» ou «agouti brown» (brun dun ou brun agouti), est causée par l'action combinée d'un facteur pour le brun : $G$ dominant $g$ (le rouge) et du facteur agouti : $A$ dominant le noir. Pour autant qu'on puisse en juger par les écrits suivants de cet auteur, BERGE $(1961 \mathrm{~b}, 1965), A$ correspondrait à un allèle au locus Agouti responsable du dun (sorte de patron zoné déjà isolé par Wilson, 1909 en race Highland) et le brun, allèle du rouge $g$, serait à un locus qui n'a pas de correspondance parmi les loci de l'homologie mais dont la création a pu être inspirée par la nomenclature allemande évoquée ci-avant.

Ces deux formules (l'allemande et la norvégienne) sortant carrément du cadre homologique, nous avions (LAUverGNe, 1966 a) proposé la formule

$$
A^{w} A^{w} \quad B^{+} B^{+} \quad C^{+} C^{+} \quad E^{+} E^{+}
$$

avec, donc, un seul variant muté : $A^{w}$ au locus $A g o u t i$, nommé d'après la ressemblance apparente avec le variant white belly de la souris.

\section{C. - Critique de la dernière formule proposée et élaboration d'une nouvelle hypothèse}

L'explication du brun ou gris brun de la Brune des Alpes par un allèle du type $A^{w}$ (white belly) de la souris implique que la nuance provient de la présence au sein d'un même poil et en alternance de phaeomélanine (jaune) et d'eumélanine (noire puisqu'on a supposé la présence de $B^{+}$allèle non muté au locus Brun $B$ ), cf. SeArle (1968) ou Silvers (1980). Or, s'il existe bien quelques poils zonés dans le pelage de la Brune des Alpes comme le fait remarquer BERGE (1961 a), la plupart des poils sont uniformément colorés et, point important qui sera mis en évidence par Misuraca et al. (1974), ne portent que de l'eumélanine, à l'exclusion de toute trace de phaeomélanine. L'explication de la couleur par un mélange de pigment est donc à rejeter et par suite, celle de l'homologie avec un allèle de type $A^{w}$.

Cette couleur anormale pour un animal uniquement eumélanique est alors explicable de deux façons:

1) par l'existence d'un facteur de dilution du type «dilution maltaise » (cf. SEARLE, 1968) ;

ou 
2) par l'existence d'un mutant $B$ (Brun) qui transforme l'eumélanine noire en eumélanine brune.

La première de ces deux hypothèses (dilution maltaise) est assez plausible à première vue car les nuances des $\mathrm{F}_{1}$ Simmental ou Charolais par races noires, mesurées par LAUVERgNe (1966 b) avec un atlas coloré, recouvrent celles qui caractérisent la race Brune des Alpes. Toutefois, cette hypothèse ne semble pas acceptable car, dans ses croisements avec les races noires ou rouges, la Brune des Alpes ne donne pas de phénotypes dilués. Il s'agit semble-t-il, simplement d'un phénomène de mimique, courant en génétique des facteurs à effets visibles.

La seconde hypothèse (un allèle en $B$ ) est beaucoup plus acceptable. Il se trouve en effet que certaines nuances des phénotypes induits à coup sûr par des allèles mutés au locus $B$ chez le mouton (Denis et al., 1978) ou la chèvre (LAUvergne \& Howell, 1978) sont les mêmes que celles en Brune des Alpes mesurées par LAUVERGNE (1966 b).

On est ainsi amené à postuler pour la Brune des Alpes la formule $B^{s} B^{s}$ au locus $B$ avec $s$ pour schwyz, une appellation courante de la race en France, qui rappelle son origine helvétique.

En ce qui concerne maintenant le locus Agouti où on ne peut placer un allèle de type $A^{w}$, comme nous l'avons vu, il faut postuler l'existence d'un allèle induisant un eumélanisme presque total, à l'exception d'éclaircissement sur les oreilles, la raie dorsale, les parties déclives et le tour du mufle. Cet allèle pourrait être nommé $A^{i}$ : raie de mulet inversée (1).

La formule nouvellement proposée pour la Brune des Alpes serait alors :

$$
A^{i} A^{i} B^{s} B^{s}
$$

pour les 2 loci Agouti et Brun après omission de la formule aux autres loci supposés porteurs d'allèles non mutés.

Il ne reste plus qu'à étayer cette hypothèse en apportant des justificatifs biochimiques et colorimétriques et en analysant les ségrégations dans des croisements où la race intervient.

\section{III. - Résultats}

\section{A. - La nature biochimique du pigment du pelage de la race Brune des Alpes}

Le test utilisé par Misuraca et al. (1974) consistait à rechercher les trichochromes indicateurs des phaeomélanines dans des extraits de poils traités par la Soude à $0,1 \mathrm{~N}$. $\mathrm{Si}$ ces extraits étaient incolores, ils ne contenaient pas de phaeomélanines.

L'analyse du pelage de races témoins noires ou rouges - les premières génétiquement eumélaniques, les secondes phaeomélaniques - a montré la validité du test qui, appliqué à la Brune des Alpes, a indiqué que celle-ci était uniquement eumélanique de même que ses croisements avec la Piémontaise ou la Frisonne Pie noir.

(1) La raie dite de mulet est en effet une raie dorsale noire sur fond rouge ou fauve plus ou moins clair. 


\section{B. - Les tests colorimétriques}

Pour mesurer la couleur du pelage nous avons utilisé l'atlas Müller (cf. LAUvergne, 1966 b). La couleur spectrale se situait dans l'orange et le rouge : 8 à 14 pour les bovins, ovins et caprins mesurés respectivement par LaUvergne (1966 b), Denis et al. (1978) et LaUVERGNe \& Howell (1978).
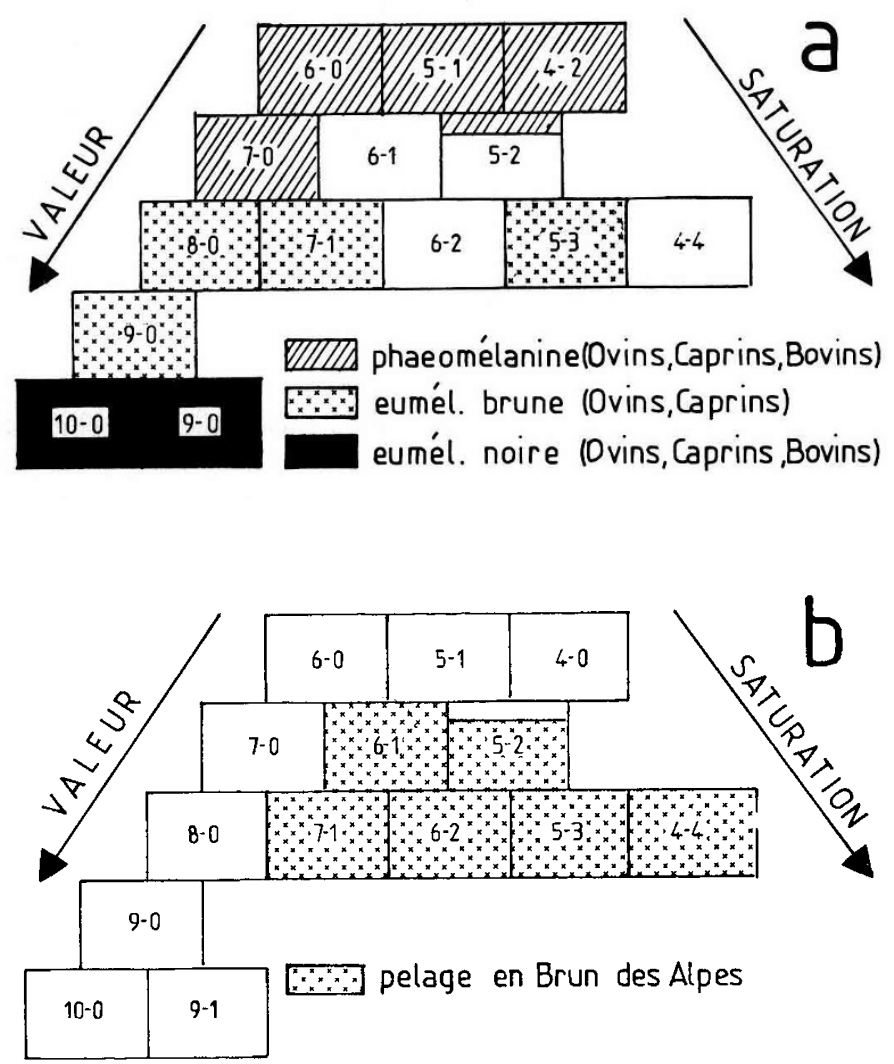

FIG. 2

a) Répartition dans le plan valeur/saturation de l'atlas Müller (cf. LAUvERGNe, 1966 b) des nuances de la phaeomélanine et de l'eumélanine noire chez les ovins, les caprins et les bovins et de l'eumélanine brune chez les ovins, et les caprins d'après LAUVERGNe (1966 b), Denis et al. (1978), LAUVERGNe \& Howell (1978) et des observations non publiées de l'auteur en race ovine Karakačan de Bulgarie.

b) Répartition, dans le plan valeur/saturation (comme en a) des nuances du pelage de 30 bovins Bruns des Alpes (18 âgés de 1 à 6 mois et 12 femelles adultes) mesurées par LAUVERGNE (1966 b).

a) Repartition, in the value/saturation level, of the Müller atlas (cf. LAUVERGNe, $1966 \mathrm{~b}$ ) of phaeomelanine and black eumelanin shades in Sheep, Goat and Cattle coat and of brown eumelanin in Sheep and Goat, according LaUvergne (1966 b), Denis et al. (1978), Lauvergne \& Howell (1979) and some unpublished data by author in Karakačan Sheep of Bulgaria.

b) Repartition in the same value/saturation (level as in a) of the coat shades of $30 \mathrm{Alp}$ brown cattle (18 between 1 and 6 months and 12 cows) measured by LAUVERGNE (1966 b). 
Dans le plan valeur/saturation les nuances observées sur le pelage de 319 bovins par Lauvergne (1966 b) se localisaient dans 16 des 55 loges numérotées $\mathrm{x}-\mathrm{y}: \mathrm{x}$ pour la valeur (de 0 à 9), y pour la saturation (de 0 à 9 également) et une comparaison avait permis de représenter dans ce plan valeur-saturation, les différents groupes : rouge foncé, rouge clair, jaune blanc, gris foncé, gris clair. Il y avait en outre l'échelle des gris (sans nuance spectrale) de 0.0 (blanc pur) à 10.0 (tout noir).

Dix-huit des trente bovins Brun des Alpes se classaient parmi les gris clair, les autres douze parmi les gris foncé. Les croisés Charolais $\times$ Frison Pie noir se répartissaient en cinq gris clair et sept foncés et les croisés Simmental $\times$ Angus ou Galloway se retrouvaient uniquement dans la catégorie gris foncé : 22 dans les mêmes nuances que les Bruns des Alpes et 12 dans les nuances que l'on ne retrouve pas en Brun des Alpes.

La représentation dans le plan valeur/saturation a été repris dans la figure 2 pour comparer les nuances des chèvres et moutons porteurs du génotype brun, et mesurés par Denis et al. (1978) et LaUvergne \& Howell (1978) (fig. 2 a) avec les bovins Bruns des Alpes mesurés par LaUVERGNe (1966 b) (fig. 2 b).

On note l'assez bon recouvrement dans la gamme des gris clair (selon la définition de Lauvergne, 1966 b). On ne retrouve apparemment pas chez la Brune des Alpes le brun foncé correspondant à l'un des deux allèles pour le brun qui semble isolable chez les chèvres.

\section{C. - Le phénotype «raie de mulet inversée» des bovins}

Si ce phénotype (pelage eumélanique avec raie dorsale claire) n'existe au standard coloré d'aucune race européenne moderne à standard univoque (sauf chez la Brune des Alpes que nous étudions ici), par contre, au cours d'examens non encore publiés, nous l'avons retrouvé dans certaines races. On a résumé ces observations dans le tableau 2.

\section{TABLEAU 2}

Phénotypes "raie de mulet inversée » observés par l'auteur dans différentes races "Reversed mule stripe» phenotypes in various breeds, authors personnal observations

\begin{tabular}{|c|c|c|c|c|}
\hline $\mathbf{N}^{\circ}$ & $\begin{array}{l}\text { Race ou } \\
\text { population }\end{array}$ & $\begin{array}{c}\text { Lieu } \\
\text { d'examen }\end{array}$ & $\begin{array}{c}\text { Date } \\
\text { d'examen }\end{array}$ & $\begin{array}{c}\text { Description du phénotype « raie de mulet » } \\
\text { observé }\end{array}$ \\
\hline 1 & $\begin{array}{c}\text { Sarde } \\
\text { autochtone }\end{array}$ & Sardaigne & 1974 & $\begin{array}{l}\text { Fond noir, anneau de museau à nuance } \\
\text { claire, raie dorsale rouge, intérieur des } \\
\text { oreilles rouge, extrémité du toupillon } \\
\text { rouge }\end{array}$ \\
\hline 2 & $\begin{array}{l}\text { Corse } \\
\text { autochtone }\end{array}$ & Corse & 1975 & idem \\
\hline 3 & Hérens & $\begin{array}{l}\text { Valais } \\
\text { (Suisse) }\end{array}$ & 1981 & idem \\
\hline 4 & Rhodope & Bulgarie & 1981 & idem \\
\hline 5 & Rhodope & Bulgarie & 1981 & $\begin{array}{l}\text { idem mais la raie dorsale et l'intérieur des } \\
\text { oreilles sont de couleur claire }\end{array}$ \\
\hline 6 & Iskar & Bulgarie & 1980 & idem $n^{0} 1$ \\
\hline
\end{tabular}


Dans la figure $1 \mathrm{~b}$ on donne la photo d'un bovin de race Corse autochtone présentant ce phénotype sur fond eumélanique noir.

Par ailleurs MAJESKIE (1970) décrit formellement ce patron en race HolsteinFriesian des Etats-Unis et, toujours dans cette contrée, OLsson (1975) le retrouve chez des croisés Chianine $\times$ Brown Swiss et Jersey $\times$ Angus.

\section{D. - Les données de croisements}

\section{1. $F_{1}$ Brun des Alpes $\times$ races rouges}

Ils sont décrits par une série d'auteurs allemands: SpanN (1913), Richardsen (1914), Staffe (1925), Wiethe-Körprich (1952). Ces auteurs notent la présence presque constante de raie de mulet inversée, d'oreilles claires et de tour du museau clair, et l'existence d'une teinte juvénile qui fonce avec l'âge pour devenir brun noirâtre à un an. Les quelques gris plus clair qui apparaissent peuvent être dus à la présence, pas toujours signalée, de rouge dilué type Fleckvieh. Les observations de MAJESKIE (1970) en race Holstein-Friesian concordent.

Les quelques observations que nous avons pu faire de tels croisements montrent qu'il faut sans doute tenir compte d'une répartition de la couleur qui présente une certaine analogie avec le patron blaireau : encolure, ventre, cuisses quasiment noirs, cependant que le flanc présente une couleur rougeâtre, en forme de croissant, en outre la raie de mulet inversée restait bien marquée ainsi que l'anneau autour du mufle et les éclaircissements des oreilles et de l'intérieur des membres.

\section{2. $F_{1}$ Brun des Alpes $\times$ fauve à extrémités noires}

Nous avons noté des animaux noirs à raie de mulet inversée mais aussi des intermédiaires entre le noir à raie de mulet inversée et le blaireau fauve.

\section{3. $F_{1}$ Brun des Alpes $\times$ races noires}

Les auteurs allemands: Henneberg (1914), Richardsen (1914), Staffe (1925), Kliesch \& Horst (1952) ainsi que Bushnell (1940), aux Etats-Unis, font état d'un phénotype quasiment noir chez l'adulte, après une phase juvénile plus claire. Dans certains cas on note des réminiscences atténuées de la raie du mulet inversée, cependant que les quelques observations où BushnelL (1940) fait mention de phénotypes plus clairs, s'expliqueraient par l'hétérozygotie (pour le rouge) de certaines femelles Holstein. On retomberait alors sur des phénotypes décrits en D.1.

\section{Les back cross}

Ils sont peu nombreux au total.

a) $B d A$ (1) $\times(B d a \times$ races rouges $)$. STAFFE (1925) note 1 noir uniforme, 1 [Brun des Alpes] (2).

b) $B d A \times(B d A \times$ races noires $)$. Les données rassemblées de StafFE \& BUSHNELL (1940) font état de 2 noir uniforme, 2 [Brun des Alpes] et 2 intermédiaires.

(1) BdA pour Brun des Alpes.

(2) La notation [Brun des Alpes] signifie : de phénotype Brun des Alpes. 


\section{IV. - Discussion}

Les observations du tableau 1 confortées par les travaux américains confirment la réalité du patron « raie de mulet inversée ». Chez les bovins : il apparaît sur n'importe quel fond eumélanique : noir ou brun.

L'interprétation du phénotype croisé Brun des Alpes $\times$ races rouges est relativement facile avec la formule proposée ici pour le Brun des Alpes: $A^{i} A^{i} B^{\star} B^{s}$ et celle des races rouges donnée par LAUVERGNe (1978 a) : $A^{r} A^{r} B^{+} B^{+}$. Il suffit d'admettre que $B+$ domine $B^{s}$ pour donner de l'eumélanine noire et que $A^{i} A^{r}$ donne un phénotype intermédiaire entre le presque complètement eumélanique $\left[\boldsymbol{A}^{i}\right]$ et le presque complètement phaeomélanique $\left[\boldsymbol{A}^{r}\right]$ : c'est tout d'abord les hésitations de la pigmentation juvénile puis, à l'âge adulte, des phénotypes qui rappellent le blaireau, avec une eumélanisation de la tête, du cou, du ventre, cependant qu'un croissant plus rouge persiste sur le haut du flanc. A noter que les auteurs américains MAJESKIE et Olsson interprètent le «blaireau» (blackisch pattern) comme induit par l'allèle $B \mathrm{p}_{\mathrm{M}}$ au locus $B \mathrm{p}$.

Une explication très semblable est valable semble-t-il, pour les $F_{1}$ Brun des Alpes $\times$ fauve à extrémités noires.

Les back cross examinés ( 2 au total) sont trop peu nombreux pour infirmer ou confirmer l'hypothèse. L'animal décrit comme noir par STAFFe (1925) étant de plus susceptible de présenter des traces du patron « raie de mulet inversée » que cet auteur cernait assez mal à l'époque.

Pour expliquer maintenant le phénotype des croisés Bruns des Alpes $\times$ races noires, compte tenu de la dominance au moins partielle des allèles plus eumélanisés au locus Agouti sur ceux qui les sont moins, on peut être tenté de revenir à l'hypothèse de Lauvergne (1966 a) faisant intervenir un allèle $A^{s}$ en $A g o u t i$ au lieu de $E^{d}$ : noir dominant au locus d'Extension (LAUVERGNe, 1977). Ce comportement de dominance semble particulier aux bovins (et peut-être au buffle africain, cf. LAUVERGNe \& RENVoISÉ, 1980), il s'oppose à la majorité des comportements de dominance en Agouti dans les espèces revues par SEARLE (1968), mais il semble que l'on doive en envisager la possibilité.

Dans ce cas-là, la $\mathrm{F}_{1}$ aurait alors pour formule $A^{s} A^{i} B^{+} B^{s}$ qui doit tout naturellement donner un phénotype quasiment eumélanique noir : $\left[A^{8}\right]$ et $\left[A^{i}\right]$ étant finalement assez proche et $B^{+}$pour l'eumélanine noire dominant $B^{s}$.

En back cross on retrouvera naturellement des noirs et des bruns à côté de nombreux phénotypes comme "raie de mulet inversée sur fond noir $A^{i} A^{i} B+B^{8}$ (peut-être l'intermédiaire noté par Bushnell, 1940), le $4^{\circ}$ génotype attendu étant $A^{8} A^{i} B^{8} B^{8}$ sans doute assez facile à confondre avec le Brun des Alpes.

Quoiqu'il en soit, la discussion tourne court vu la rareté et l'imprécision des données actuellement disponibles. Des études plus approfondies doivent être développées : 
- pour identifier histologiquement et biochimiquement les divers pigments mélaniques impliquée ;

- pour décrire phénotypiquement avec précision les différents patrons observés en les observant depuis la naissance jusqu'à l'âge adulte.

\section{V. - Conclusion}

Malgré les imprécisions qui la caractérisent, cette étude entraîne un certain nombre de modifications dans les séries alléliques Agouti $A$, Brun $B$ et Extension E.

Au locus Agouti la modification la plus vraisemblable est l'introduction du nouvel allèle $A^{i}$ (raie de mulet inversée) cependant que la réintroduction de $A^{s}$ s'inscrit dans la logique du comportement de dominance des allèles de la série dans l'espèce bovine.

$\mathrm{Au}$ locus Brun l'introduction d'un allèle muté $B^{s}$ semble devoir être envisagée ; c'est une grande nouveauté chez les bovins où l'on pensait que l'allèle sauvage $B^{+}$ pour le noir n'avait jamais muté (cf. LaUvergne, 1966 a ; Searle, 1968).

Au locus d'Extension, vu la logique du comportement de dominance des allèles en Agouti, il semble inutile d'invoquer l'existence d'un allèle $E^{d}$ de noir dominant.

Il faut, pour finir, mentionner le locus de Dilution $D$, même si on n'y enregistre aucune modification de la série, à cause de la mimique nuancière entre $A^{r} A^{r} B^{+} B^{+} D^{f} D^{+}$ et $A^{i} A^{i} B^{s} B^{s} D^{+} D^{+}$.

Le nouveau «statut» encore provisoire auquel on aboutit ainsi est présenté dans la colonne 4 du tableau 1 .

Reçu pour publication en octobre 1981.

\section{Remerciements}

L'auteur a grandement profité des conseils du Dr A.G. SEARLE (Medical Research Council, Harwell, G.B.) et du $D^{r}$ B. Denis (E.N.V. Nantes).

L'examen des phénotypes en race d'Hérens a été possible grâce au concours de L. Avon (I.T.E.B., Paris).

Les données de croisements Brun des Alpes par Gascon nous ont été fournies par G. BonNES, E.N.F.A., Castanet-Tolosan.

\section{Summary}

A possible genetic formula for the coat colour of Brown Mountain cattle of Switzerland

Up to now the coat colour of Brown Mountain cattle of Switzerland was considered as induced by one allele at the Agouti locus $\left(A^{w}\right)$, the other loci, being supposed to carry wild factor specially the B locus (Brown) with an allele giving black eumelanin. This article 
proposes a new formula with $A^{i}$ (i for inverted mule stripe) in $A$ and $B^{s}$ (s for schwyz) in $B$ inducing a brown chocolate eumelanin.

This interpretation is based on observations of inverted mule stripe pattern on black eumelanin background in other breeds and on the similar color of Swiss Brown body and that of other Ruminants showing a true chocolate brown phenotype. This is the first time that a mutant for chocolate-brown eumelanin at $B$ locus has been proposed in cattle.

\section{Références bibliographiques}

Adalsteinsson S., 1970. Colour inheritance in Icelandic sheep and relation between colour, fertility and fertilization. J. agr. Res. Icel., 2, 3-135.

Adalsteinsson S., 1974. Colour inheritance in farm animals and its application in selection. Irst. World Congr. Genet. appl. Livest. Prod., 1, 29-37.

Baldwin C.S., Gilmore N.S., FechHeimer N.S., Rife D.C., 1956. Inheritance of black hair patterns in cattle lacking the extension factor for black (E). II - Blackish pattern. J. Dairy Sci., 39, 435-439.

Berge S., 1949. Inheritance of dun, brown and brindle colour in cattle. Heredity, 3, 195-204. BERGE S., 1961 a. Influence of dun on brown and brindle in cattle. Z. Tierz. ZüchtBiol., 75, 298-306.

BERGe S., 1961 b. Arv av farge hos storfe. Institutt for husdyravl, Norges Landbrukshəgskole Vollebekk, Norge, $69 \mathrm{p}$.

BERgE S., 1965. Storfegarger. Norges Landbrukshəgskole, Institutt for Husdyravl, Meld. $\mathrm{Nr} 201,46 \mathrm{p}$.

Bonadonna T., 1959. Le razze bovine - Buffali, Cattali, Zebú. Progresso Zootecnico, Milano, 121-220.

Bushnell R.H., 1940. Colour genes in Holstein-Friesian by Brown Swiss crosses. J. Hered., 31, 252-256.

Castle W.E., 1931. Genetics and Eugenics (4th ed.). Harvard University Press, Cambridge Mass., 474 p.

Castle W.E., 1940. Mammalian Genetics. Harvard University Press, Cambridge, Mass., $25-48,145-148$.

Denis B., Lauvergne J.J., Théret M., 1978. Un variant clair du Mouflon corsico-sarde (Ovis musimon) dû à un allèle au locus B. Ann. Génét. Sél. anim., 10, 507-515.

Engeler W., 1947. Das schweizerische Braunvieh. Huber, Frauenfeld, 30.

HaldDane J.B.S., 1927. The comparative genetics of colour in Rodents and Carnivora. Biol. Rev., 2, 199-212.

IBSEN H.L., 1933. Cattle inheritance. I. color. Genetics, 18, 441-480.

Kliesch J., Horst P., 1960. Untersuchungen zur Frage der Farbvererbung beim Rind. Z. Tierz. ZüchtBiol., 74, 106-118.

LAUVergne J.J., 1966 a. Génétique de la couleur du pelage des bovins domestiques (Bos taurus L.). Bibliogr. genet., 20, 1-68.

Lauvergne J.J., $1966 \mathrm{~b}$. Contribution à l'étude de l'hérédité de la couleur du pelage chez les bovins domestiques. Thèse $3^{\mathrm{e}}$ cycle, Fac. Sci. Univ. Paris, $58 \mathrm{p}$.

Lauvergne J.J., 1970. Mise en évidence de l'existence du phénotype noir et feu dans deux nouvelles espèces de Mammifères. Rev. roum. Biol., Sér. Zool., 15, 113-118.

Lauvergne J.J., 1977. New alleles at the Agouti locus $(A)$ in domestic Ruminants. Proc. Xth Pigment cell Conf. Boston, oct. 1977. Abstr. no. 69.

Lauvergne J.J., 1978. Gènes de coloration du pelage de chèvres Alpines Chamoisées et Poitevines. Ann. Génét. Sél. anim., 10, 181-189.

Lauvergne J.J., 1979. Homology of alleles at the Agouti locus in Ruminants. Ann. Dermatol. Vénéréol. (Paris), 106, 411. 
Lauvergne J.J, Adalsteinsson S., 1976. Gènes pour la couleur de la toison de la brebis Corse. Ann. Génét. Sél. anim., 8, 153-172.

Lauvergne J.J., Howell W.E., 1978. Un premier inventaire génétique de la chèvre Corse (gènes à effets visibles). Ethnozootechnie (22), 86-93.

Lauvergne J.J., Renvoisé C., 1980. Hérédité des variations de couleur du pelage du Buffle africain (Syncerus caffer). Ann. Génét. Sél. anim., 12, 1-7.

LitTle C.C., 1958. Coat Color Genes in Rodents and Carnivores. Rev. Biol., 33, 103-137.

MAJESKIE J.L., 1970. Characteristics and inheritance of certain coat color and patterns in cattle. Ph.D. Thesis, Kansas State University, 75 p.

Mason I.L., 1956. A dictionary of Livestock Breeds. Commonwealth Agricultural Bureaux, Farnham Royal, Bucks, England, 25, 87.

Misuraca G., Prota G., Lauvergne J.J., 1974. Pigments mélaniques du pelage de quelques races bovines : le schéma possible de certaines actions géniques. Ann. Génét. Sél. anim., 6, 399-404.

Olsson T.A., 1975. An analysis of the inheritance of coat color in cattle. Master Thesis, Iowa State University, $138 \mathrm{p}$.

OLson T.A., 1980. Choice of a wild-type Standard in Color Genetics of domestic cattle. J. Hered., 71, 442-444.

Prota G., Searle A.G., 1978. Biochemical sites of gene action for melanogenesis in Mammals. Ann. Génét. Sél. anim., 10, 1-8.

Quittet E., Denis B., 1979. Races bovines françaises. La Maison Rustique, Paris (3e éd.), 28-29, 42-43.

RENDEL J., 1957. Nedärvningen av färg och teckning hos husdjur. K. Landtbr. Akad. Handl., Stockholm, 96, 208-263.

RichaRDSEN, 1914. Vererbung der Rinderfarben bei Farbenkreuzung. Dtsch. Landw. Tierz., 18, $61-65$.

Searle A.G., 1968. Comparative Genetics of Coat Colour in Mammals. Logos Press Acad. Press, New York, London, 19, 69, 181-187.

Shrode R.R., LuSH J.L., 1946. The genetics of cattle. Advanc. Genet., 1, 209-261.

Silvers W.K., 1980. The Coat Colors of Mice. A model for Mammalian Gene Action and Interaction. Springer, New York, Heidelberg, Berlin, 6-44.

Smith A.D.B., 1924. The inheritance of Coat Colour in Cattle, with special reference to the Shorthorn breed. Master Sci. Thesis, Iowa State College.

SpanN, 1913. Kreuzungen zwischen Algäuer und Afrikaner-Rindern. Dtsch. Landw. Tierz., $17,18-20$.

StAFFe A., 1925. Hybridatavismus bei der Kreuzung rot- und schwarzbunter Hollander mit braungrauen Alpenvieh und die Verzögerung seines Aufscheinens durch Kastration. Z. Tierz. ZüchtBiol., 2, 179-203.

Wiethe-KöRPRICH M., 1952. Einige Betrachtungen über die Farbvererbung bein Rind. Z. Tierz. ZüchtBiol., 60, 205-208.

Wilson J., 1909. The colours of Highland cattle. Sci. Proc. R. Dublin Soc., 12, 66-76.

WRIGHT S., 1917. Color inheritance in Mammals. VI - Cattle. J. Hered., 8, 521-527. 